\title{
Improved Preimage Attacks on RIPEMD-160 and HAS-160
}

\author{
Yanzhao Shen ${ }^{1,2}$ and Gaoli Wang ${ }^{3}$ \\ ${ }^{1}$ School of Mathematics, Shandong University, Jinan 250100, Shandong, China \\ [e-mail: yanzhaoshen@mail.sdu.edu.cn] \\ ${ }^{2}$ Key Laboratory of Cryptologic Technology and Information Security, Ministry of Education, \\ Shandong University, Jinan 250100, Shandong, China \\ ${ }^{3}$ Shanghai Key Laboratory of Trustworthy Computing, School of Computer Science and Software Engineering, \\ East China Normal University, Shanghai 200062, China \\ [e-mail: glwang@sei.ecnu.edu.cn] \\ *Corresponding author: Gaoli Wang
}

Received August 30, 2017; accepted September 30, 2017; published February 28, 2018

\begin{abstract}
The hash function RIPEMD-160 is a worldwide ISO/IEC standard and the hash function HAS-160 is the Korean hash standard and is widely used in Korea. On the basis of differential meet-in-the-middle attack and biclique technique, a preimage attack on 34-step RIPEMD-160 with message padding and a pseudo-preimage attack on 71-step HAS-160 without message padding are proposed. The former is the first preimage attack from the first step, the latter increases the best pseudo-preimage attack from the first step by 5 steps. Furthermore, we locate the linear spaces in another message words and exchange the bicliques construction process and the mask vector search process. A preimage attack on 35-step RIPEMD-160 and a preimage attack on 71-step HAS-160 are presented. Both of the attacks are from the intermediate step and satisfy the message padding. They improve the best preimage attacks from the intermediate step on step-reduced RIPEMD-160 and HAS-160 by 4 and 3 steps respectively. As far as we know, they are the best preimage and pseudo-preimage attacks on step-reduced RIPEMD-160 and HAS-160 respectively in terms of number of steps.
\end{abstract}

Keywords: Cryptography, Preimage attack, RIPEMD-160, HAS-160, Differential meet-in-the-middle, Hash function

This work was supported by National Basic Research Program of China (973 Program) 2013CB834205, National Natural Science Foundation of China (Nos. 61373142, 61572125, 61632012) and Shanghai High-Tech Field Project of 16511101400.

This work is the extension of “(Pseudo-) Preimage Attacks on Step-Reduced HAS-160 and RIPEMD-160" which appeared in the 17th International Conference on Information Security (ISC 2014). 


\section{Introduction}

Hash Functions play an important role in modern cryptography. They should satisfy three classical security properties: collision resistance, preimage resistance and second preimage resistance. With the breakthroughs in the collision attacks on MD5 [1], SHA-0 [2] and SHA-1 [3], research on preimage attack is strongly motivated (see [4-11] for example). For an $n$-bit hash function, a generic algorithm requires $2^{n}$ computations for finding a preimage. The meet-in-the-middle attack $[4,12]$ is the basic tool used in preimage attacks which is improved by splice-and-cut technique [4], biclique technique [6], initial structure technique [10] and so on. Knellwolf and Khovratovich [7] introduced the differential meet-in-the-middle attack which is suitable for the hash functions with linear message expansion and weak diffusion properties. Recently, Espitau et al. [13] generalized it to the higher-order differential meet-in-the-middle attack.

RIPEMD-160 [14] was designed by Dobbertin et al. and standardized in ISO/ IEC 10118-3 [15]. It is one of the unbroken hash functions which implemented in numerous security protocols. The first collision attack on RIPEMD-160 was proposed by Mendel et al. [16]. Mendel et al. [17] improved the collision attack using the method of the attacks on MD5 and SHA-1 [1, 3]. They obtained a 48-step semi-free-start near-collision attack and a 36-step semi-free-start collision attack on RIPEMD-160. Later Mendel et al. [18] improved the 36-step semi-free-start collision attack to 42-step. Furthermore, they, using a carefully designed non-linear path search tool, obtained a 36-step semi-free-start collision attack from the first step. As for the preimage attack, Ohtahara et al. [8] presented the first preimage attack on step-reduced RIPEMD-160. They gave a 30-step second-preimage attack and a 31-step preimage attack on RIPEMD-160 using the local-collision and initial structure techniques. Moreover, Sasaki and Wang [19] proposed the distinguishing attacks on RIPEMD-160. They gave a practical 2-dimension sum distinguisher on 42-step RIPEMD-160 and a theoretical one on 51-step RIPEMD-160.

HAS-160 [20] is the Korean hash standard and is widely used in Korea. Its structure is similar to SHA-0 and SHA-1 expect the message expansion. Yun et al. [21] presented the first cryptanalysis on HAS-160. They proposed a collision attack on 45-step HAS-160 by applying techniques introduced by Wang et al. [22]. Later Cho et al. [23] extended the previous work to 53 steps by selecting message differences judiciously and constructing a more complicated first round differential path. Mendel and Rijmen [24] improved the attack on 53-step HAS-160 to a practical one by using a slightly different message modification technique and extended the attack to 59 steps. The best collision attack on HAS-160 is a practical 65-step semi-free-start collision attack which proposed by Mendel et al. [25]. Sasaki and Aoki [9] proposed the first preimage attack on step-reduced HAS-160. They presented a 48-step preimage attack using splice-and-cut and partial-matching techniques and a 52-step preimage attack using local-collision technique. Hong et al. [5] improved the Sasaki and Aoki's work [9] to 68 steps. Moreover, Kircanski et al. [26] proposed a boomerang attack on the full version of HAS-160.

This paper improves the preimage and pseudo-preimage attacks on step-reduced RIPEMD-160 and HAS-160 using differential meet-in-the-middle attack and biclique technique. A preimage attack on 34-step RIPEMD-160 (with message padding) with complexity $2^{158.91}$ and a pseudo-preimage attack on 71-step HAS-160 (without message padding) with complexity $2^{158.13}$ are presented. Both of the attacks are from the first step. 
Furthermore, preimage attacks from the intermediate step and satisfying the message padding are also proposed. A preimage attack on 35-step RIPEMD-160 holds with a complexity of $2^{159.38}$ and a preimage attack on 71-step HAS-160 holds with a complexity of $2^{158.97}$. In the attack on RIPEMD-160, we locate the linear spaces in one message word to decrease the error probability. Owing to the linear spaces located in the same message word and the bicliques located in the Left branch and the Right branch, the bicliques are more sophisticated. So we exchange the bicliques construction process and the mask vector search process to make the mask vector match the constant bits of initial values. For this reason, we improve the preimage attack on RIPEMD-160 from 34-step to 35-step. Because the pseudo-preimage attack on 71-step HAS-160 uses the message word $m_{15}$, it does not satisfy the message padding. However, the preimage attack on 71-step HAS-160 easily satisfies the message padding by fixing the linear spaces in message words $m_{0}, m_{10}, m_{6}$ and $m_{12}$. The dimension of the linear spaces increases from 3 to 5 , which benefits from the linear spaces located in these words. In this case, the complexity of pseudo-preimage attack is improved and a preimage attack on 71-step HAS-160 is obtained. As far as we know, they are the best preimage and pseudo-preimage attacks on step-reduced RIPEMD-160 and HAS-160 in term of number of steps. The previous work and our results about the (pseudo-)preimage attacks are summarized in Table 1.

Table 1. Summary of the (pseudo-)preimage attacks on RIPEMD-160 and HAS-160

\begin{tabular}{|c|c|c|c|c|}
\hline \multirow{2}{*}{ Hash Function } & \multirow{2}{*}{ Steps } & \multicolumn{2}{|c|}{ Complexity } & \multirow{2}{*}{ Source } \\
\cline { 3 - 4 } & & Pseudo-preimage & Preimage & \\
\hline \hline RIPEMD-160 & $31(50-79)$ & $2^{148}$ & $2^{155}$ & {$[8]$} \\
\hline RIPEMD-160 & $34(0-33)$ & $2^{155.81}$ & $2^{158.91}$ & Section 4.1 \\
\hline RIPEMD-160 & $35(1-35)$ & $2^{156.75}$ & $2^{159.38}$ & Section 4.2 \\
\hline HAS-160 & $48(1-48,21-68)$ & $2^{128}$ & $2^{145}$ & {$[9]$} \\
\hline HAS-160 & $52(12-63)$ & $2^{144}$ & $2^{153}$ & {$[9]$} \\
\hline HAS-160 & $65(0-64)$ & $2^{143.4}$ & $2^{152.7}$ & {$[5]$} \\
\hline HAS-160 & $67(0-66)$ & $2^{154}$ & $2^{158}$ & {$[5]$} \\
\hline HAS-160 & $68(12-79)$ & $2^{150.7}$ & $2^{156.3}$ & {$[5]$} \\
\hline HAS-160 & $71(0-70)$ & $2^{158.13}$ & $2^{158.97}$ & Section 4.3 \\
\hline HAS-160 & $71(6-76)$ & $2^{155.93}$ & & Section 4.4 \\
\hline
\end{tabular}

Section 2 summarizes the related techniques and differential meet-in-the-middle attack with bicliques. Section 3 gives a brief description of RIPEMD-160 and HAS-160. Section 4 presents preimage and pseudo-preimage attacks on step-reduced RIPEMD-160 and HAS-160. Finally, we conclude the paper in Section 5.

\section{Preliminary}

In this part, we briefly summarize the differential meet-in-the-middle attack and the biclique search algorithm. Here, some notations are introduced in advance(see Table 2).

Table 2. The major symbols used in this paper

\begin{tabular}{|c|c|}
\hline Symbols & Definitions \\
\hline \hline$L D_{1}$ & the linear space used in $C F_{1}$ \\
\hline$L D_{2}$ & the linear space used in $C F_{2}$ \\
\hline$d$ & the dimension of $L D_{1}$ and $L D_{2}$ \\
\hline$n$ & the length of the chaining value and hash value \\
\hline
\end{tabular}




\begin{tabular}{|c|c|}
\hline$P$ & the output list of $C F_{3}$ \\
\hline$Q$ & the input list of $C F_{3}$ \\
\hline$P\left[\delta_{2}\right]$ & the element of $P$ corresponding to $\delta_{2}$ \\
\hline$Q\left[\delta_{1}\right]$ & the element of $Q$ corresponding to $\delta_{1}$ \\
\hline$T$ & the mask vector, where $T \in\{0,1\}^{n}$ is a $d$ dimension vector \\
\hline$\Delta[i]$ & the $i$-th bit of $\Delta$, where $0 \leq i \leq n-1$ \\
\hline
\end{tabular}

\subsection{Converting pseudo-preimages to a preimage}

The classical algorithm that converts pseudo-preimages to a preimage [27] is described. Assume there is an algorithm that finds a pseudo-preimage in the complexity of $2^{k}$. Prepare a table that includes $2^{n-k / 2}$ entries of pseudo-preimages. The complexity of this step is $2^{n+k / 2}=2^{n-k / 2} \times 2^{k}$. Randomly chosen $2^{n+k / 2}$ 1-block message, calculate the output of the message, then one of them agrees with the entries in the table with high probability. The complexity of the matching step is $2^{n+k / 2}$. The corresponding message is a preimage of the hash function. Therefore, the total complexity for finding a preimage is $2^{n+k / 2+1}=2^{n+k / 2}+2^{n+k / 2}$.

\subsection{The differential meet-in-the-middle attack}

The differential meet-in-the-middle technique interprets the meet-in-the-middle attack in the differential view. Combined with the statistical tool, it can analyse more steps of hash functions. The differential meet-in-the-middle attack with bicliques is summarized as follows (see also Fig. 1).

- Split the compression function and construct the linear spaces. Split the compression function $C F$ into 3 parts, $C F=C F_{3} \bullet C F_{2} \bullet C F_{1}$. Choose $L D_{1}$ and $L D_{2}$ such that $L D_{1} \cap L D_{2}=\{0\}$.

- Construct the bicliques in $\boldsymbol{C F}_{3}$. For all $\left(\delta_{1}, \delta_{2}\right) \in L D_{1} \times L D_{2}$, using the biclique search algorithm constructs the bicliques such that $P\left[\delta_{2}\right]=C F_{3}\left(M \oplus \delta_{1} \oplus \delta_{2}, Q\left[\delta_{1}\right]\right)$.

- Search for the mask vector $T$. Randomly choose $M$ and $Q[0]$, compute $P=C F_{3}(M, Q[0])$. For each $\delta_{1} \in L D_{1}$ and $\delta_{2} \in L D_{2}$, search for the difference $\Delta_{1}$ and $\Delta_{2}$, such that $\Delta_{1}=C F_{1}(M, P[0]) \oplus C F_{1}\left(M \oplus \delta_{1}, P[0]\right)$ and $\Delta_{2}=C F_{2}^{-1}(M, Q[0]) \oplus$ $C F_{2}^{-1}\left(M \oplus \delta_{2}, Q[0]\right)$ hold with high probability. Then for each $\left(\delta_{1}, \delta_{2}\right) \in L D_{1} \times L D_{2}$, compute $\Delta=C F_{1}\left(M \oplus \delta_{1}, P[0]\right) \oplus \Delta_{1} \oplus C F_{2}^{-1}\left(M \oplus \delta_{2}, Q[0]\right) \oplus \Delta_{2}$. For each bit $i$ in $\Delta$, count the number of $\Delta[i]=1$. Set those $d$ bits of $T$ to 1 which have the lowest counters and the other bits of $T$ to 0 .

- $\quad$ Search for the candidate preimage. Compute the list $L_{1}=C F_{1}\left(M \oplus \delta_{2}, P\left[\delta_{2}\right]\right) \oplus \Delta_{2}$ and the list $L_{2}=C F_{2}^{-1}\left(M \oplus \delta_{1}, Q\left[\delta_{1}\right]\right) \oplus \Delta_{1}$. If $L_{1}=_{T} L_{2}$, then $M \oplus \delta_{1} \oplus \delta_{2}$ is a candidate pseudo-preimage. For a mask vector $T \in\{0,1\}^{n}, L_{1}=_{T} L_{2}$ means $T \wedge\left(L_{1} \oplus L_{2}\right)=0$, where $\wedge$ denotes bitwise AND.

The error is defined as: $M \oplus \delta_{1} \oplus \delta_{2}$ is a preimage, but we reject it as false. Assume $\bar{\alpha}$ is the the error probability, $\Gamma$ is the cost of $C F_{1}$ and $C F_{2}$, and $\Gamma_{r e}$ is the cost of retesting a candidate 
preimage. The total complexity of the differential meet-in-the-middle preimage attack is $2^{n-d} \times\left(\Gamma+\Gamma_{r e}\right) /(1-\bar{\alpha})$.

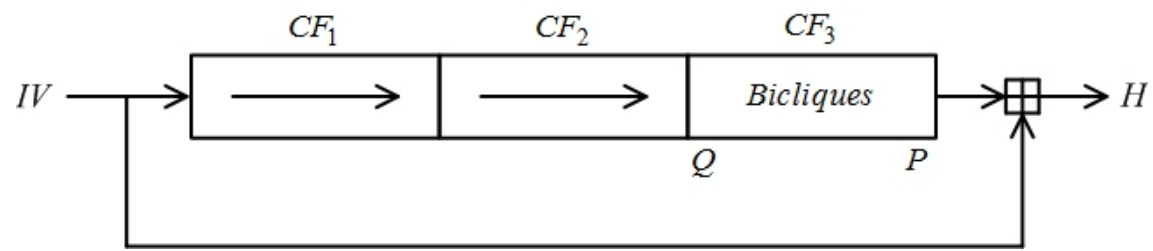

Fig. 1. Schematic view of the differential meet-in-the-middle attack with bicliques

\subsection{The biclique search algorithm}

A biclique for $C F_{3}$ is a tuple $\left\{M, L D_{1}, L D_{2}, Q, P\right\}$, where $M$ is a message, such that for all $\left(\delta_{1}, \delta_{2}\right) \in L D_{1} \times L D_{2}, P\left[\delta_{2}\right]=C F_{3}\left(M \oplus \delta_{1} \oplus \delta_{2}, Q\left[\delta_{1}\right]\right)$. Assume $\overline{C F_{3}}$ is linearized by $C F_{3}$, i.e., replacing + by $\oplus$ and setting the constants to 0 . The bicliques can be searched as follows.

- Linearize $C F_{3}$ and split $\overline{C F_{3}}$ into 2 parts, $\overline{C F_{3}}=\overline{C F_{3}^{2}} \bullet \overline{C F_{3}^{1}}$. Assume $F T\left(\delta_{2}\right)$ is the differences of the outputs of $\overline{C F_{3}^{1}}$ corresponding to $\delta_{2}$, and $B T\left(\delta_{1}\right)$ is the differences of the outputs of $\overline{\left(\mathrm{CF}_{3}^{2}\right)^{-1}}$ corresponding to $\delta_{1}$.

- For each $\delta_{1} \in L D_{1}$ and $\delta_{2} \in L D_{2}$ calculate $B T\left(\delta_{1}\right)$ and $F T\left(\delta_{2}\right)$, such that $B T\left(\delta_{1}\right)=\overline{\left(C F_{3}^{2}\right)^{-1}}\left(\delta_{1}, 0\right)$ and $F T\left(\delta_{2}\right)=\overline{C F_{3}^{1}}\left(\delta_{2}, 0\right)$, deduce the sufficient conditions to make sure the output differences of all non-linear Boolean functions are zero.

- Assume $C V$ is the intermediate chaining value which connects $C F_{3}^{1}$ and $C F_{3}^{2}$. Randomly choose $M$ and $C V$, set a part of the sufficient conditions.

- For each $\delta_{1} \in L D_{1}$ and $\delta_{2} \in L D_{2}$, calculate $Q\left[\delta_{1}\right]$ and $P\left[\delta_{2}\right]$, such that $Q\left[\delta_{1}\right]=\left(C F_{3}^{1}\right)^{-1}\left(M \oplus \delta_{2}, C V \oplus F T\left(\delta_{2}\right) \oplus B T\left(\delta_{1}\right)\right)$ holds for every $\delta_{2} \in L D_{2}$ and $P\left[\delta_{2}\right]=C F_{3}^{2}\left(M \oplus \delta_{1}, C V \oplus F T\left(\delta_{2}\right) \oplus B T\left(\delta_{1}\right)\right)$ holds for every $\delta_{1} \in L D_{1}$.

- If $\left\{M, L D_{1}, L D_{2}, Q, P\right\}$ constitute a biclique, return $(M, Q[0])$. Otherwise, repeat the above steps until a biclique is constructed.

\section{Description of RIPEMD-160 and HAS-160}

In this section, we briefly introduce our targeted hash functions. Both of the hash functions adopt the Merkel-Damgård structure and process 512-bit input message blocks and produce a 160-bit hash value. They first call the message padding procedure. The message padding procedure ensures the padded message is a multiple of 512 bits. For an l-bit message, append the bit " 1 " to the end of the message, followed by $k$ " 0 " bits, where $k$ is the smallest non-negative solution to the equation $l+k+1 \equiv 448 \bmod 512$. Then append the 64-bit block that is equal to the number $l$ expressed using a binary representation. The words $m_{14}$ and $m_{15}$ in the last message block represent the message length. Then, using the compression function, they update the initial value and produce the hash value. The compression function of RIPEMD-160 and HAS-160 consist of a message expansion function and a state update transformation, see the details in [14] and [20]. 


\subsection{Description of RIPEMD-160}

In this part, the compression function of RIPEMD-160 is described.

\subsubsection{Message Expansion}

The message expansion of RIPEMD-160 splits the 512-bit message block $M$ into 16 words $m_{0}$, $\ldots, m_{15}$, and expands them into 160 expanded message words $w_{i}^{L}$ and $w_{i}^{R}(0 \leq i \leq 79)$. The expansion is specified in Table 2.

Table 2. Message order and rotation of RIPEMD-160

\begin{tabular}{|c|c|c|c|c|c|c|c|c|c|c|c|c|c|c|c|c|c|c|c|c|}
\hline$i$ & 0 & 1 & 2 & 3 & 4 & 5 & 6 & 7 & 8 & 9 & 10 & 11 & 12 & 13 & 14 & 15 & 16 & 17 & 18 & 19 \\
\hline$w_{i}{ }^{2}$ & 0 & 1 & 2 & 3 & 4 & 5 & 6 & 7 & 8 & 9 & 10 & 11 & 12 & 13 & 14 & 15 & 7 & 4 & 13 & 1 \\
\hline$w_{i}^{R}$ & 5 & 14 & 7 & 0 & 9 & 2 & 11 & 4 & 13 & 6 & 15 & 8 & 1 & 10 & 3 & 12 & 6 & 11 & 3 & 7 \\
\hline$s_{i}^{L}$ & 11 & 14 & 15 & 12 & 5 & 8 & 7 & 9 & 11 & 13 & 14 & 15 & 6 & 7 & 9 & 8 & 7 & 6 & 8 & 13 \\
\hline$s_{i}^{R}$ & 8 & 9 & 9 & 11 & 13 & 15 & 15 & 5 & 7 & 7 & 8 & 11 & 14 & 14 & 12 & 6 & 9 & 13 & 15 & 7 \\
\hline \hline$i$ & 20 & 21 & 22 & 23 & 24 & 25 & 26 & 27 & 28 & 29 & 30 & 31 & 32 & 33 & 34 & 35 & 36 & 37 & 38 & 39 \\
\hline$w_{i}^{L}$ & 10 & 6 & 15 & 3 & 12 & 0 & 9 & 5 & 2 & 14 & 11 & 8 & 3 & 10 & 14 & 4 & 9 & 15 & 8 & 1 \\
\hline$w_{i}^{R}$ & 0 & 13 & 5 & 10 & 14 & 15 & 8 & 12 & 4 & 9 & 1 & 2 & 15 & 5 & 1 & 3 & 7 & 14 & 6 & 9 \\
\hline$s_{i}^{L}$ & 11 & 9 & 7 & 15 & 7 & 12 & 15 & 9 & 11 & 7 & 13 & 12 & 11 & 13 & 6 & 7 & 14 & 9 & 13 & 15 \\
\hline$s_{i}^{R}$ & 12 & 8 & 9 & 11 & 7 & 7 & 12 & 7 & 6 & 15 & 13 & 11 & 9 & 7 & 15 & 11 & 8 & 6 & 6 & 14 \\
\hline \hline$i$ & 40 & 41 & 42 & 43 & 44 & 45 & 46 & 47 & 48 & 49 & 50 & 51 & 52 & 53 & 54 & 55 & 56 & 57 & 58 & 59 \\
\hline$w_{i}^{L}$ & 2 & 7 & 0 & 6 & 13 & 11 & 5 & 12 & 1 & 9 & 11 & 10 & 0 & 8 & 12 & 4 & 13 & 3 & 7 & 15 \\
\hline$w_{i}^{R}$ & 11 & 8 & 12 & 2 & 10 & 0 & 4 & 13 & 8 & 6 & 4 & 1 & 3 & 11 & 15 & 0 & 5 & 12 & 2 & 13 \\
\hline$s_{i}^{L}$ & 14 & 8 & 13 & 6 & 5 & 12 & 7 & 5 & 11 & 12 & 14 & 15 & 14 & 15 & 9 & 8 & 9 & 14 & 5 & 6 \\
\hline$S_{i}^{R}$ & 12 & 13 & 5 & 14 & 13 & 13 & 7 & 5 & 15 & 5 & 8 & 11 & 14 & 14 & 6 & 14 & 6 & 9 & 12 & 9 \\
\hline \hline$i$ & 60 & 61 & 62 & 63 & 64 & 65 & 66 & 67 & 68 & 69 & 70 & 71 & 72 & 73 & 74 & 75 & 76 & 77 & 78 & 79 \\
\hline$w_{i}^{L}$ & 14 & 5 & 6 & 2 & 4 & 0 & 5 & 9 & 7 & 12 & 2 & 10 & 14 & 1 & 3 & 8 & 11 & 6 & 15 & 13 \\
\hline$w_{i}^{R}$ & 9 & 7 & 10 & 14 & 12 & 15 & 10 & 4 & 1 & 5 & 8 & 7 & 6 & 2 & 13 & 14 & 0 & 3 & 9 & 11 \\
\hline$s_{i}^{L}$ & 8 & 6 & 5 & 12 & 9 & 15 & 5 & 11 & 6 & 8 & 13 & 12 & 5 & 12 & 13 & 14 & 11 & 8 & 5 & 6 \\
\hline$s_{i}^{R}$ & 12 & 5 & 15 & 8 & 8 & 5 & 12 & 9 & 12 & 5 & 14 & 6 & 8 & 13 & 6 & 5 & 15 & 13 & 11 & 11 \\
\hline
\end{tabular}

\subsubsection{State Update Transformation}

The state update transformation of RIPEMD-160 starts from an initial value of five 32-bit words $A_{0}, B_{0}, C_{0}, D_{0}, E_{0}$ and updates them in 80 steps. For the $i$-th $(0 \leq i \leq 79)$ step, the 32-bit words $w_{i}^{L}$ and $w_{i}^{R}$ are used and the state variables $A_{i}^{L}, B_{i}^{L}, C_{i}^{L}, D_{i}^{L}, E_{i}^{L}, A_{i}^{R}, B_{i}^{R}$, $C_{i}^{R}, D_{i}^{R}, E_{i}^{R}$ are update as:

Left branch:

$$
\begin{aligned}
& B_{i+1}^{L}=\left(A_{i}^{L}+F_{i}^{L}\left(B_{i}^{L}, C_{i}^{L}, D_{i}^{L}\right)+w_{i}^{L}+k_{i}^{L}\right)<<<s_{i}^{L}+E_{i}^{L}, \\
& A_{i+1}^{L}=E_{i}^{L}, C_{i+1}^{L}=B_{i}^{L}, D_{i+1}^{L}=C_{i}^{L} \ll<<10, E_{i+1}^{L}=D_{i}^{L} .
\end{aligned}
$$

Right branch:

$$
\begin{aligned}
& B_{i+1}^{R}=\left(A_{i}^{R}+F_{i}^{R}\left(B_{i}^{R}, C_{i}^{R}, D_{i}^{R}\right)+w_{i}^{R}+k_{i}^{R}\right)<<<s_{i}^{R}+E_{i}^{R}, \\
& A_{i+1}^{R}=E_{i}^{R}, C_{i+1}^{R}=B_{i}^{R}, D_{i+1}^{R}=C_{i}^{R}<<<10, E_{i+1}^{R}=D_{i}^{R} .
\end{aligned}
$$

The bitwise Boolean function $F_{i}^{L}\left(B_{i}^{L}, C_{i}^{L}, D_{i}^{L}\right), F_{i}^{R}\left(B_{i}^{R}, C_{i}^{R}, D_{i}^{R}\right)$, round constant $k_{i}^{L}$, $k_{i}^{R}$ are given in Table 3 and the rotational constant $s_{i}^{L}, s_{i}^{R}$ are specified in Table 2.

If $M$ is the last block, $\left(B_{0}+C_{80}^{L}+D_{80}^{R}, C_{0}+D_{80}^{L}+E_{80}^{R}, D_{0}+E_{80}^{L}+A_{80}^{R}, E_{0}+A_{80}^{L}+B_{80}^{R}\right.$, $A_{0}+B_{80}^{L}+C_{80}^{R}$ ) is the hash value, otherwise as the inputs of the next message block. 
Table 3. The Boolean function and round constant of RIPEMD-160

\begin{tabular}{|c|c|c|c|c|}
\hline Step & $F_{i}^{L}\left(B_{i}^{L}, C_{i}^{L}, D_{i}^{L}\right)$ & $\boldsymbol{k}_{i}^{L}$ & $F_{i}^{R}\left(B_{i}^{R}, C_{i}^{R}, D_{i}^{R}\right)$ & $k_{i}^{R}$ \\
\hline $0 \leq i \leq 15$ & $B_{i}^{L} \oplus C_{i}^{L} \oplus D_{i}^{L}$ & 0x00000000 & $B_{i}^{R} \oplus\left(C_{i}^{R} \vee\left(\neg D_{i}^{R}\right)\right)$ & 0x50a28be6 \\
\hline $16 \leq i \leq 31$ & $\left(B_{i}^{L} \wedge C_{i}^{L}\right) \vee\left(\left(\neg B_{i}^{L}\right) \wedge D_{i}^{L}\right)$ & 0x5a827999 & $\left(B_{i}^{R} \wedge D_{i}^{R}\right) \vee\left(C_{i}^{R} \wedge\left(\neg D_{i}^{R}\right)\right)$ & 0x5c4dd124 \\
\hline $32 \leq i \leq 47$ & $\left(B_{i}^{L} \vee\left(\neg C_{i}^{L}\right)\right) \oplus D_{i}^{L}$ & 0x6ed9eba1 & $\left(B_{i}^{R} \vee\left(\neg C_{i}^{R}\right)\right) \oplus D_{i}^{R}$ & 0x6d703ef3 \\
\hline $48 \leq i \leq 63$ & $\left(B_{i}^{L} \wedge D_{i}^{L}\right) \vee\left(C_{i}^{L} \wedge\left(\neg D_{i}^{L}\right)\right)$ & 0x8f1bbcdc & $\left(B_{i}^{R} \wedge C_{i}^{R}\right) \vee\left(\left(\neg B_{i}^{R}\right) \wedge D_{i}^{R}\right)$ & 0x7a6d76e9 \\
\hline $64 \leq i \leq 79$ & $B_{i}^{L} \oplus\left(C_{i}^{L} \vee\left(\neg D_{i}^{L}\right)\right)$ & 0xa953fd4e & $B_{i}^{R} \oplus C_{i}^{R} \oplus D_{i}^{R}$ & $0 \mathrm{x} 00000000$ \\
\hline
\end{tabular}

\subsection{Description of HAS-160}

In this part, the compression function of HAS-160 is described.

\subsubsection{Message Expansion}

The message expansion of HAS-160 splits the 512-bit message block $M$ into 16 words $m_{0}$, $\ldots, m_{15}$, and expands them into 80 expanded message words $w_{i}(0 \leq i \leq 79)$. The expansion is specified in Table 4.

Table 4. Message expansion of HAS-160

\begin{tabular}{|c|c|c|c|c|c|c|c|c|c|c|}
\hline$i$ & 0 & 1 & 2 & 3 & 4 & 5 & 6 & 7 & 8 & 9 \\
\hline$w_{i}$ & $m_{8} \oplus m_{9} \oplus m_{10} \oplus m_{11}$ & $m_{15}$ & $m_{15}$ & $m_{15}$ & $m_{15}$ & $m_{12} \oplus m_{13} \oplus m_{14} \oplus m_{15}$ & $m_{4}$ & $m_{5}$ & $m_{6}$ & $m_{7}$ \\
\hline \hline$i$ & 10 & 11 & 12 & 13 & 14 & 15 & 16 & 17 & 18 & 19 \\
\hline$w_{i}$ & $m_{0} \oplus m_{1} \oplus m_{2} \oplus m_{3}$ & $m_{8}$ & $m_{9}$ & $m_{10}$ & $m_{11}$ & $m_{4} \oplus m_{5} \oplus m_{6} \oplus m_{7}$ & $m_{12}$ & $m_{13}$ & $m_{14}$ & $m_{15}$ \\
\hline \hline$i$ & 20 & 21 & 22 & 23 & 24 & 25 & 26 & 27 & 28 & 29 \\
\hline$w_{i}$ & $m_{11} \oplus m_{14} \oplus m_{1} \oplus m_{4}$ & $m_{3}$ & $m_{6}$ & $m_{9}$ & $m_{12}$ & $m_{7} \oplus m_{10} \oplus m_{13} \oplus m_{0}$ & $m_{15}$ & $m_{2}$ & $m_{5}$ & $m_{8}$ \\
\hline \hline$i$ & 30 & 31 & 32 & 33 & 34 & 35 & 36 & 37 & 38 & 39 \\
\hline$w_{i}$ & $m_{3} \oplus m_{6} \oplus m_{9} \oplus m_{12}$ & $m_{11}$ & $m_{14}$ & $m_{1}$ & $m_{4}$ & $m_{15} \oplus m_{2} \oplus m_{5} \oplus m_{8}$ & $m_{7}$ & $m_{10}$ & $m_{13}$ & $m_{0}$ \\
\hline \hline$i$ & 40 & 41 & 42 & 43 & 44 & 45 & 46 & 47 & 48 & 49 \\
\hline$w_{i}$ & $m_{4} \oplus m_{13} \oplus m_{6} \oplus m_{15}$ & $m_{12}$ & $m_{5}$ & $m_{14}$ & $m_{7}$ & $m_{8} \oplus m_{1} \oplus m_{10} \oplus m_{3}$ & $m_{0}$ & $m_{9}$ & $m_{2}$ & $m_{11}$ \\
\hline \hline$i$ & 50 & 51 & 52 & 53 & 54 & 55 & 56 & 57 & 58 & 59 \\
\hline$w_{i}$ & $m_{12} \oplus m_{5} \oplus m_{14} \oplus m_{7}$ & $m_{4}$ & $m_{13}$ & $m_{6}$ & $m_{15}$ & $m_{0} \oplus m_{9} \oplus m_{2} \oplus m_{11}$ & $m_{8}$ & $m_{1}$ & $m_{10}$ & $m_{3}$ \\
\hline \hline$i$ & 60 & 61 & 62 & 63 & 64 & 65 & 66 & 67 & 68 & 69 \\
\hline$w_{i}$ & $m_{15} \oplus m_{10} \oplus m_{5} \oplus m_{0}$ & $m_{7}$ & $m_{2}$ & $m_{13}$ & $m_{8}$ & $m_{11} \oplus m_{6} \oplus m_{1} \oplus m_{12}$ & $m_{3}$ & $m_{14}$ & $m_{9}$ & $m_{4}$ \\
\hline \hline$i$ & 70 & 71 & 72 & 73 & 74 & 75 & 76 & 77 & 78 & 79 \\
\hline$w_{i}$ & $m_{7} \oplus m_{2} \oplus m_{13} \oplus m_{8}$ & $m_{15}$ & $m_{10}$ & $m_{5}$ & $m_{0}$ & $m_{3} \oplus m_{14} \oplus m_{9} \oplus m_{4}$ & $m_{11}$ & $m_{6}$ & $m_{1}$ & $m_{12}$ \\
\hline
\end{tabular}

\subsubsection{State Update Transformation}

The state update transformation of HAS-160 starts from an initial value of five 32-bit words $A_{0}, B_{0}, C_{0}, D_{0}, E_{0}$ and updates them in 80 steps. For the $i$-th $(0 \leq i \leq 79)$ step, the 32-bit word $w_{i}$ is used and the state variables $A_{i}, B_{i}, C_{i}, D_{i}, E_{i}$ are update as:

$$
\begin{aligned}
& A_{i+1}=\left(A_{i}<<<s_{i}^{1}\right)+F_{i}\left(B_{i}, C_{i}, D_{i}\right)+E_{i}+w_{i}+k_{i}, \\
& B_{i+1}=A_{i}, C_{i+1}=B_{i}<<<s_{i}^{2}, D_{i+1}=C_{i}, E_{i+1}=D_{i} .
\end{aligned}
$$

The bitwise Boolean function $F_{i}\left(B_{i}, C_{i}, D_{i}\right)$, round constant $k_{i}$ and rotation const $s_{i}^{2}$ used in each step are given in Table 5 . The rotational constant $s_{i}^{1}$ are given in Table 6. 
Table 5. The Boolean function and round constant of HAS-160

\begin{tabular}{|c|c|c|c|}
\hline Step & $\boldsymbol{F}_{\boldsymbol{i}}\left(\boldsymbol{B}_{\boldsymbol{i}}, \boldsymbol{C}_{\boldsymbol{i}}, \boldsymbol{D}_{\boldsymbol{i}}\right)$ & $\boldsymbol{k}_{\boldsymbol{i}}$ & $\boldsymbol{s}_{\boldsymbol{i}}{ }^{\mathbf{2}}$ \\
\hline \hline $0 \leq i \leq 19$ & $\left(B_{i} \wedge C_{i}\right) \vee\left(\left(\neg B_{i}\right) \wedge D_{i}\right)$ & $0 x 00000000$ & 10 \\
\hline $20 \leq i \leq 39$ & $B_{i} \oplus C_{i} \oplus D_{i}$ & $0 \times 5 a 827999$ & 17 \\
\hline $40 \leq i \leq 59$ & $C_{i} \oplus\left(B_{i} \vee\left(\neg D_{i}\right)\right)$ & $0 x 6 e d 9 e b a 1$ & 25 \\
\hline $60 \leq i \leq 79$ & $B_{i} \oplus C_{i} \oplus D_{i}$ & $0 x 8 f 1 b b c d c$ & 30 \\
\hline
\end{tabular}

Table 6. The shift $s_{i}^{1}$ of HAS-160

\begin{tabular}{|c|c|c|c|c|c|c|c|c|c|c|c|c|c|c|c|c|c|c|c|c|}
\hline Step & \multicolumn{10}{|c|}{$\boldsymbol{s}_{\boldsymbol{i}}^{\mathbf{1}}$} \\
\hline \hline$i \bmod 20$ & 5 & 11 & 7 & 15 & 6 & 13 & 8 & 14 & 7 & 12 & 9 & 11 & 8 & 15 & 6 & 12 & 9 & 14 & 5 & 13 \\
\hline
\end{tabular}

If $M$ is the last block, ( $\left.A_{80}+A_{0}, B_{80}+B_{0}, C_{80}+C_{0}, D_{80}+D_{0}, E_{80}+E_{0}\right)$ is the hash value, otherwise as the inputs of the next message block.

\section{Attacks on step-reduced RIPEMD-160 and HAS-160}

In this section, we describe the details of the preimage and pseudo-preimage attacks on step-reduced RIPEMD-160 and HAS-160. Assume $\Delta m_{i}=(a, b)$ means the bits from the $a$-th bit to the $b$-th bit of $\Delta m_{i}$ take all possible values, and the other bits are $0 . \Delta m_{i}$ can be represented by a linear space. $\overline{C F_{1}}$ and $\overline{C F_{2}^{-1}}$ are obtained from $C F_{1}$ and $C F_{2}^{-1}$ respectively by linearizing the step function, i.e., replacing + by $\oplus$ and setting the constants and input chaining values of $C F_{1}$ and $C F_{2}^{-1}$ to 0 .

\subsection{Preimage attack on 34-step RIPEMD-160 from the first step}

\subsubsection{Split the compression function and construct the linear spaces}

The forward direction $C F_{1}$ is from step 1 to step 33 in left branch connecting with the step of computing the hash value (marked with red dotted box in Fig. 2) and the linear space of this part is $L D_{1}=\left\{\Delta m_{0}\|\ldots\| \Delta m_{15} \mid \Delta m_{0}=(27,31), \Delta m_{i}=0,1 \leq i \leq 15\right\}$. The basis of $L D_{1}$ is as follows:

Basis 1:

0x08000000, 0x00000000, 0x00000000, 0x00000000, 0x00000000, 0x00000000, 0x00000000, 0x00000000, 0x00000000, 0x00000000, 0x00000000, 0x00000000, 0x00000000, 0x00000000, 0x00000000, 0x00000000

Basis 2:

0x10000000, 0x00000000, 0x00000000, 0x00000000, 0x00000000, 0x00000000, 0x00000000, 0x00000000, 0x00000000, 0x00000000, 0x00000000, 0x00000000, 0x00000000, 0x00000000, 0x00000000, 0x00000000

Basis 3:

0x20000000, 0x00000000, 0x00000000, 0x00000000, 0x00000000, 0x00000000, 0x00000000, 0x00000000, 0x00000000, 0x00000000, 0x00000000, 0x00000000, 0x00000000, 0x00000000, 0x00000000, 0x00000000 
Basis 4:

0x40000000, 0x00000000, 0x00000000, 0x00000000, 0x00000000, 0x00000000, 0x00000000, 0x00000000, 0x00000000, 0x00000000, 0x00000000, 0x00000000, 0x00000000, 0x00000000, 0x00000000, 0x00000000

Basis 5:

0x80000000, 0x00000000, 0x00000000, 0x00000000, 0x00000000, 0x00000000, 0x00000000, 0x00000000, 0x00000000, 0x00000000, 0x00000000, 0x00000000, 0x00000000, 0x00000000, 0x00000000, 0x00000000

Let $C V_{i}{ }^{L}$ and $C V_{i}^{R}$ denote the inputs of the $i$-th step of left branch and right branch respectively. Then the difference corresponding to $C V_{25}^{L}$ of $C F_{1}\left(M \oplus \delta_{1}, C V_{1}^{L}\right)$ and $C F_{1}\left(M, C V_{1}^{L}\right)$ is always zero. The backward direction $C F_{2}^{-1}$ is from step 6 to step 33 in right branch and the linear space is $L D_{2}=\left\{\Delta m_{0}\|\ldots\| \Delta m_{15} \mid \Delta m_{2}=(4,8), \Delta m_{i}=0,0 \leq i \leq 15, i \neq 2\right\}$. The basis of $L D_{2}$ is as follows:

Basis 1:

0x00000000, 0x00000000, 0x00000010, 0x00000000, 0x00000000, 0x00000000, 0x00000000, 0x00000000, 0x00000000, 0x00000000, 0x00000000, 0x00000000, 0x00000000, 0x00000000, 0x00000000, 0x00000000

Basis 2:

0x00000000, 0x00000000, 0x00000020, 0x00000000, 0x00000000, 0x00000000, 0x00000000, 0x00000000, 0x00000000, 0x00000000, 0x00000000, 0x00000000, 0x00000000, 0x00000000, 0x00000000, 0x00000000

Basis 3:

0x00000000, 0x00000000, 0x00000040, 0x00000000, 0x00000000, 0x00000000, 0x00000000, 0x00000000, 0x00000000, 0x00000000, 0x00000000, 0x00000000, 0x00000000, 0x00000000, 0x00000000, 0x00000000

Basis 4:

0x00000000, 0x00000000, 0x00000080, 0x00000000, 0x00000000, 0x00000000, 0x00000000, 0x00000000, 0x00000000, 0x00000000, 0x00000000, 0x00000000, 0x00000000, 0x00000000, 0x00000000, 0x00000000

Basis 5:

0x00000000, 0x00000000, 0x00000100, 0x00000000, 0x00000000, 0x00000000, 0x00000000, 0x00000000, 0x00000000, 0x00000000, 0x00000000, 0x00000000, 0x00000000, 0x00000000, 0x00000000, 0x00000000

The difference corresponding to $C V_{31}^{R}$ of $C F_{2}^{-1}\left(M \oplus \delta_{2}, C V_{6}^{R}\right)$ and $C F_{2}^{-1}\left(M, C V_{6}^{R}\right)$ is always zero. The biclique $C F_{3}$ covers step 0 in left branch connecting with step 0 to step 5 in right branch. It is noted that the initial value $I V$ is an intermediate value in the bicliques, and $I V$ also affects the last step (marked with red dotted box in Fig. 2), so the truncation mask 
vector can only be chosen in a smaller scope, e.g., the unchanged bits of $I V$. Therefore, we choose the linear spaces $L D_{1}$ and $L D_{2}$ as above so that we can get a proper truncation mask vector to make sure the error probability of the attack is as small as possible. The schematic view of the preimage attack on 34-step RIPEMD-160 is shown in Fig. 2.

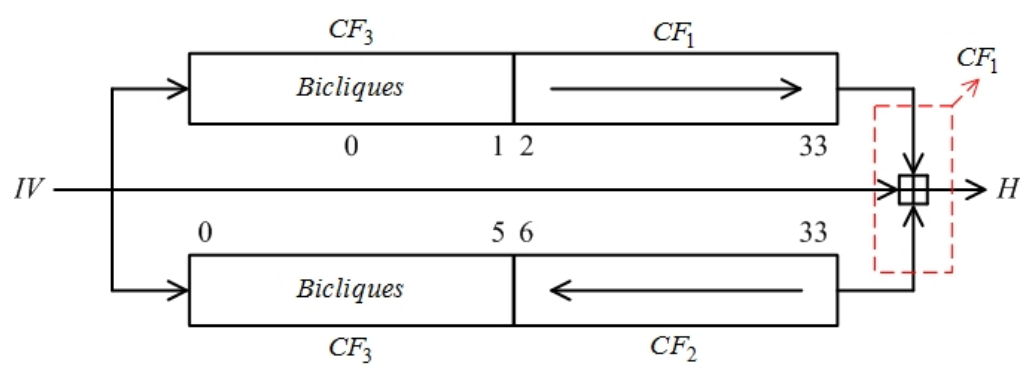

Fig. 2. Schematic view of the preimage attack on 34-step RIPEMD-160

\subsubsection{Construct 3.5-step bicliques}

The bicliques which are constructed for $C_{3}$ cover step 5 to step 0 in right branch connecting with step 0 in left branch (which equals to 3.5 steps), such that for all $\left(\delta_{1}, \delta_{2}\right) \in L D_{1} \times L D_{2}$, $C V_{1}^{L}\left[\delta_{2}\right]=C F_{3}\left(M \oplus \delta_{1} \oplus \delta_{2}, C V_{6}^{R}\left[\delta_{1}\right]\right)$. The parameters used in Section 2.3 are set as follows: $C F_{3}^{1}$ covers step 0 in left branch connecting with step 0 to step 2 in right branch and $C F_{3}^{2}$ covers step 3 to step 5 in right branch, $C V=\left(A_{3}^{R}, B_{3}^{R}, C_{3}^{R}, D_{3}^{R}, E_{3}^{R}\right)$. For $\delta_{2} \in L D_{2}$ and $\delta_{1} \in L D_{1}$, we can deduce a set of sufficient conditions that make sure $F T\left(\delta_{2}\right)$ and $B T\left(\delta_{1}\right)$ hold. Then the bicliques can be constructed by using the biclique search algorithm in Section 2.3. Note that in the biclique search algorithm, we only set the sufficient conditions in steps 1 , 2 and 3 in right branch. By setting those conditions, a biclique can be obtained in a few seconds on a PC. So the complexity of constructing a biclique is negligible. An example of the 3.5-step bicliques is shown in Table 7.

Table 7. An example of 3.5-step bicliques in RIPEMD-160

\begin{tabular}{|c|c|c|c|c|c|c|c|}
\hline word & $m_{0}$ & & $m_{2}$ & $m_{5}$ & $m_{7}$ & $m_{9}$ & $m_{14}$ \\
\hline value & 0x07b30181 & & 0x5effd80e & 0xf7962189 & 0xff1152bb & 0xa9bc41d8 & 0x00000000 \\
\hline \hline$C V_{6}{ }^{R}$ & $A$ & & $B$ & $C$ & $D$ & $E$ & \\
\hline value & 0x8fcfef67 & & 0xc592715e & 0x26d8dac7 & 0x71f4450e & 0x6c6740a0 & \\
\hline
\end{tabular}

\subsubsection{Search for the mask vector and estimate the error probability}

The mask vector is $T_{34}=(0,0 x f 80,0,0,0)$. By extensive experiments, the total test number is $2^{26}$ and the error probability is about 0.331 . The test algorithm is the same as Knellwolf and Khovratovich's Algorithm 3 [7].

\subsubsection{The attack procedure}

When the bicliques are obtained, we can get $C V_{1}^{L}\left[\delta_{2}\right]$ and $C V_{6}^{R}\left[\delta_{1}\right]$, then compute $L_{1}=C F_{1}\left(M \oplus \delta_{2}, C V_{1}^{L}\left[\delta_{2}\right]\right) \oplus \Delta_{2}$ and $L_{2}=C F_{2}^{-1}\left(M \oplus \delta_{1}, C V_{6}^{R}\left[\delta_{1}\right]\right) \oplus \Delta_{1} . \Delta_{1}=\overline{C F_{1}}\left(\delta_{1}, 0\right)$ and $\Delta_{2}=\overline{C F_{2}^{-1}}\left(\delta_{2}, 0\right)$, where $C V_{1}^{L}, C V_{6}^{R}, k_{i}^{L}$ and $k_{i}^{R}$ are 0 . We need to calculate 6 steps 
(step 25 to step 33 in left branch add step 31 to step 33 in right branch, which equals to $6=(9+3) / 2$ steps $)$ to retest a candidate pseudo-preimage, $\Gamma_{r e}=6 \Gamma / 34$, and the complexity of constructing a biclique is negligible. Thus, the complexity of the pseudo-preimage attack is $2^{155.81} \approx 2^{160-5} \times(1+6 / 34) /(1-0.331)$. Furthermore, this attack can be converted to a preimage attack ( 2 message blocks, with padding) with complexity $2^{158.91}$.

\subsection{Preimage attack on 35-step RIPEMD-160 from the second step}

\subsubsection{Split the compression function and construct the linear spaces}

The forward direction $C F_{1}$ is from step 3 to step 35 in left branch connecting with the step of computing the hash value (marked with red dotted box in Fig. 3 ) and the linear space of this part is $L D_{1}=\left\{\Delta m_{0}\|\ldots\| \Delta m_{15} \mid \Delta m_{2}=(27,31), \Delta m_{i}=0,0 \leq i \leq 15, i \neq 2\right\}$. The basis of $L D_{1}$ is as follows:

Basis 1:

0x00000000, 0x00000000, 0x08000000, 0x00000000, 0x00000000, 0x00000000, 0x00000000, 0x00000000, 0x00000000, 0x00000000, 0x00000000, 0x00000000, 0x00000000, 0x00000000, 0x00000000, 0x00000000

Basis 2:

0x00000000, 0x00000000, 0x10000000, 0x00000000, 0x00000000, 0x00000000, 0x00000000, 0x00000000, 0x00000000, 0x00000000, 0x00000000, 0x00000000, 0x00000000, 0x00000000, 0x00000000, 0x00000000

Basis 3:

0x00000000, 0x00000000, 0x20000000, 0x00000000, 0x00000000, 0x00000000, 0x00000000, 0x00000000, 0x00000000, 0x00000000, 0x00000000, 0x00000000, Basis 4: 0x00000000, 0x00000000, 0x00000000, 0x00000000

0x00000000, 0x00000000, 0x40000000, 0x00000000, 0x00000000, 0x00000000, 0x00000000, 0x00000000, 0x00000000, 0x00000000, 0x00000000, 0x00000000, 0x00000000, 0x00000000, 0x00000000, 0x00000000

Basis 5:

0x00000000, 0x00000000, 0x80000000, 0x00000000, 0x00000000, 0x00000000, 0x00000000, 0x00000000, 0x00000000, 0x00000000, 0x00000000, 0x00000000, 0x00000000, 0x00000000, 0x00000000, 0x00000000

The difference corresponding to $C V_{28}^{L}$ of $C F_{1}\left(M \oplus \delta_{1}, C V_{3}^{L}\right)$ and $C F_{1}\left(M, C V_{3}^{L}\right)$ is always zero. The backward direction $C F_{2}^{-1}$ is from step 6 to step 35 in right branch and the linear space of this part is $L D_{2}=\left\{\Delta m_{0}\|\ldots\| \Delta m_{15} \mid \Delta m_{2}=(13,17), \Delta m_{i}=0,0 \leq i \leq 15, i \neq 2\right\}$. The basis of $L D_{2}$ is as follows: 
Basis 1:

0x00000000, 0x00000000, 0x00002000, 0x00000000, 0x00000000, 0x00000000, 0x00000000, 0x00000000, 0x00000000, 0x00000000, 0x00000000, 0x00000000, 0x00000000, 0x00000000, 0x00000000, 0x00000000

Basis 2:

0x00000000, 0x00000000, 0x00004000, 0x00000000, 0x00000000, 0x00000000, 0x00000000, 0x00000000, 0x00000000, 0x00000000, 0x00000000, 0x00000000, 0x00000000, 0x00000000, 0x00000000, 0x00000000

Basis 3:

0x00000000, 0x00000000, 0x00008000, 0x00000000, 0x00000000, 0x00000000, 0x00000000, 0x00000000, 0x00000000, 0x00000000, 0x00000000, 0x00000000, 0x00000000, 0x00000000, 0x00000000, 0x00000000

Basis 4:

0x00000000, 0x00000000, 0x00010000, 0x00000000, 0x00000000, 0x00000000, 0x00000000, 0x00000000, 0x00000000, 0x00000000, 0x00000000, 0x00000000, 0x00000000, 0x00000000, 0x00000000, 0x00000000

Basis 5:

0x00000000, 0x00000000, 0x00020000, 0x00000000, 0x00000000, 0x00000000, 0x00000000, 0x00000000, 0x00000000, 0x00000000, 0x00000000, 0x00000000, 0x00000000, 0x00000000, 0x00000000, 0x00000000

The difference corresponding to $C V_{31}^{R}$ of $C F_{2}^{-1}\left(M \oplus \delta_{2}, C V_{6}^{R}\right)$ and $C F_{2}^{-1}\left(M, C V_{6}^{R}\right)$ is always zero. The biclique $C F_{3}$ covers step 1 and step 2 in left branch connecting with step 1 to step 5 in right branch. Because the initial value is an intermediate value in the bicliques and affects the last step (marked with red dotted box in Fig. 3), the mask vector can only be chosen in a small scope, e.g., the unchanged least significant bits of the initial value. Therefore, we choose the linear spaces $L D_{1}$ and $L D_{2}$ as above so that we can get a proper mask vector to make the error probability as low as possible. The schematic view of the preimage attack on 35-step RIPEMD-160 is shown in Fig. 3.

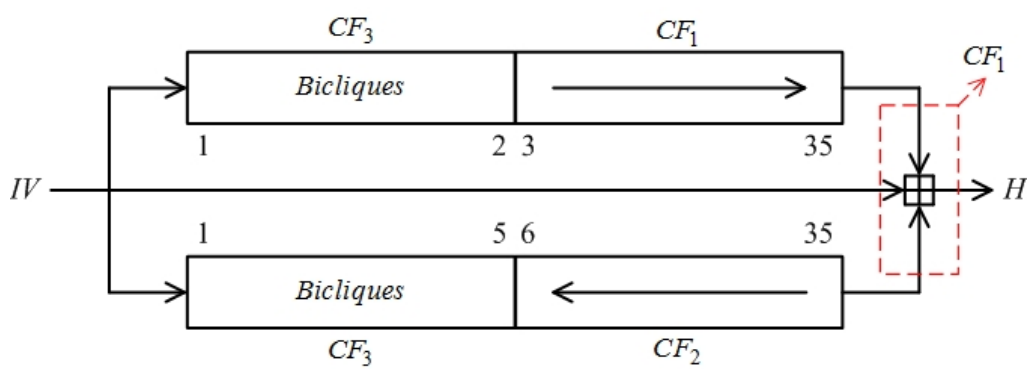

Fig. 3. Schematic view of the preimage attack on 35-step RIPEMD-160 


\subsubsection{Construct 3.5-step bicliques}

The bicliques which are constructed for $C_{3}$ cover step 5 to step 1 in right branch connecting with step 1 to step 2 in left branch (which equals to 3.5 steps), such that for all $\left(\delta_{1}, \delta_{2}\right) \in L D_{1} \times L D_{2}, C V_{3}^{L}\left[\delta_{2}\right]=C F_{3}\left(M \oplus \delta_{1} \oplus \delta_{2}, C V_{6}^{R}\left[\delta_{1}\right]\right)$. The parameters used in Section 2.3 are set as follows: $C F_{3}^{1}$ covers step 5 to step 3 in right branch and $C F_{3}^{2}$ covers step 2 to step 1 in right branch connecting with step 1 to step 2 in left branch, $C V=\left(A_{3}^{R}, B_{3}^{R}, C_{3}^{R}, D_{3}^{R}, E_{3}^{R}\right) . F T\left(\delta_{2}\right)$ is deduced as follows:

$$
\begin{aligned}
& A_{3}^{R} \wedge(0 \mathrm{x} 3 \mathrm{e} 00000)=\left(\left(\left(m_{2} \oplus \delta_{2}\right) \wedge(0 \times 3 \mathrm{e} 000)\right) \oplus(0 \times 3 \mathrm{e} 000)\right)<<8, \\
& D_{3}^{R} \wedge(0 \mathrm{x} 3 \mathrm{e} 000)=\left(\left(m_{2} \oplus \delta_{2}\right) \wedge(0 \times 3 \mathrm{e} 000)\right) \oplus(0 \times 3 \mathrm{e} 000), \\
& E_{3}^{R} \wedge(0 \mathrm{x} 1 \mathrm{f})=\left(\left(m_{2} \oplus \delta_{2}\right) \wedge(0 \times 3 \mathrm{e} 000)\right)<<<19 .
\end{aligned}
$$

Condition that satisfies $F T\left(\delta_{2}\right)$ is $C_{3}^{R} \wedge(0 \times 3 \mathrm{e} 000)=0 \times 3 \mathrm{e} 000 . B T\left(\delta_{1}\right)$ is deduced as follows:

$$
\begin{aligned}
& B_{3}^{R} \wedge(0 \mathrm{x} 8000000 \mathrm{f})=\left(\left(\left(m_{2} \oplus \delta_{1}\right) \wedge(0 \mathrm{xf8000000)}) \oplus(0 \mathrm{xf8000000)})<<<4,\right.\right. \\
& C_{3}^{R} \wedge(0 \mathrm{x} 7 \mathrm{c} 00000)=\left(\left(m_{2} \oplus \delta_{1}\right) \wedge(0 \mathrm{xf8000000)})<<<27,\right. \\
& C_{3}^{R} \wedge(0 \mathrm{xf8000000)})=\left(\left(m_{2} \oplus \delta_{1}\right) \wedge(0 \mathrm{xf8000000)}) \oplus(0 \mathrm{xf} 8000000) .\right.
\end{aligned}
$$

Conditions that satisfy $B T\left(\delta_{1}\right)$ are $A_{3}^{R} \wedge(0 \mathrm{x} 8000000 \mathrm{f})=0, B_{3}^{R} \wedge(0 \mathrm{xf} 8)=0 \mathrm{xf} 8, E_{3}^{R} \wedge$ $(0 x f f c 00000)=0 x f f c 0000$.

By setting those conditions, a biclique example can be obtained less than 1 second on a PC. So the complexity of constructing a biclique is negligible. An example of the 3.5-step biclique is shown in Table 8. Note that to satisfy the padding process, $M_{15}=0 x 3 b f$ (the message length is $959=512+447$ ) and the least significant bit of $M_{13}$ is set to 1 .

Table 8. An example of 3.5-step bicliques in RIPEMD-160

\begin{tabular}{|c|c|c|c|c|c|c|}
\hline word & $m_{0}$ & $m_{1}$ & $m_{2}$ & $m_{7}$ & $m_{9}$ & $m_{14}$ \\
\hline value & 0x74a2aec5 & 0xe84269fa & 0xeef5d697 & 0x4e5f2844 & 0x7e98f8a9 & 0x00000000 \\
\hline \hline$C V_{6}{ }^{R}$ & $A$ & $B$ & $C$ & $D$ & $E$ & \\
\hline value & 0x8fbcac5d & 0x140846d5 & 0xdf46e46e & 0x18115673 & 0xee7be4c7 & \\
\hline
\end{tabular}

\subsubsection{Search for the mask vector and estimate the error probability}

One can check that the 4 least significant bits of $D_{1}^{R}$ and 2 least significant bits of $E_{1}^{R}$ are unchanged in bicliques and the error probability in these bits are acceptable. So the mask vector is $T_{35}=(0 \times f, 0 \times 2,0,0,0)$. By extensive experiments, the total test number is $2^{30}$ and the error probability is about 0.65 .

\subsubsection{The attack procedure}

When the bicliques are obtained, we can get $C V_{3}^{L}\left[\delta_{2}\right]$ and $C V_{6}^{R}\left[\delta_{1}\right]$, then compute $L_{1}=C F_{1}\left(M \oplus \delta_{2}, C V_{3}^{L}\left[\delta_{2}\right]\right) \oplus \Delta_{2}$ and $L_{2}=C F_{2}^{-1}\left(M \oplus \delta_{1}, C V_{6}^{R}\left[\delta_{1}\right]\right) \oplus \Delta_{1} . \Delta_{1}=\overline{C F_{1}}\left(\delta_{1}, 0\right)$ and $\Delta_{2}=\overline{C F_{2}^{-1}}\left(\delta_{2}, 0\right)$, where $C V_{3}^{L}, C V_{6}^{R}, k_{i}^{L}$ and $k_{i}^{R}$ are 0 . We need to calculate 6.5 steps (step 28 to step 35 in left branch add step 31 to step 35 in right branch, which equals to $6.5=(8+5) / 2$ steps $)$ to retest a candidate pseudo-preimage, $\Gamma_{r e}=6.5 \Gamma / 35$, and the 
complexity of constructing a biclique is negligible. Thus, the complexity of the pseudo-preimage attack is $2^{156.75} \approx 2^{160-5} \times(1+6.5 / 35) /(1-0.65)$. Furthermore, this attack can be converted to a preimage attack ( 2 message blocks, with padding) with complexity $2^{159.38}$.

\subsection{Pseudo-preimage attack on 71-step HAS-160 from the first step}

\subsubsection{Split the compression function and construct the linear spaces}

The forward direction $C F_{1}$ is from step 59 to step 70 connecting with step 0 to step 20 and the linear space of this part is $L D_{1}=\left\{\Delta m_{0}\|\ldots\| \Delta m_{15} \mid \Delta m_{10}=\Delta m_{11}=\Delta m_{12}=\Delta m_{15}=(26,28)\right.$, $\left.\Delta m_{i}=0,0 \leq i \leq 15, i \neq 10,11,12,15\right\}$. The basis of $L D_{1}$ is as follows:

Basis 1:

0x00000000, 0x00000000, 0x00000000, 0x00000000, 0x00000000, 0x00000000, 0x00000000, 0x00000000, 0x00000000, 0x00000000, 0x04000000, 0x04000000, 0x04000000, 0x00000000, 0x00000000, 0x04000000

Basis 2:

0x00000000, 0x00000000, 0x00000000, 0x00000000, 0x00000000, 0x00000000, 0x00000000, 0x00000000, 0x00000000, 0x00000000, 0x08000000, 0x08000000, 0x08000000, 0x00000000, 0x00000000, 0x08000000

Basis 3:

0x00000000, 0x00000000, 0x00000000, 0x00000000, 0x00000000, 0x00000000, 0x00000000, 0x00000000, 0x00000000, 0x00000000, 0x10000000, 0x10000000, 0x10000000, 0x00000000, 0x00000000, 0x10000000

Let $C V_{i}$ denote the input of the $i$-th step of HAS-160. In this case, the input difference of the 13-th step of $C F_{1}\left(M \oplus \delta_{1}, C V_{59}\right)$ and $C F_{1}\left(M, C V_{59}\right)$ is zero. The backward direction $C F_{2}^{-1}$ is from step 52 to step 21 and the linear space of this part is $L D_{2}=\left\{\Delta m_{0}\|\ldots\| \Delta m_{15} \mid \Delta m_{3}=\Delta m_{6}=\Delta m_{8}=\Delta m_{15}=(29,31), \Delta m_{i}=0,0 \leq i \leq 15, i \neq 3,6,8,15\right\} \quad$. The basis of $L D_{2}$ is as follows:

Basis 1:

0x00000000, 0x00000000, 0x00000000, 0x20000000, 0x00000000, 0x00000000, 0x20000000, 0x00000000, 0x20000000, 0x00000000, 0x00000000, 0x00000000, 0x00000000, 0x00000000, 0x00000000, 0x20000000

Basis 2:

0x00000000, 0x00000000, 0x00000000, 0x40000000, 0x00000000, 0x00000000, 0x40000000, 0x00000000, 0x40000000, 0x00000000, 0x00000000, 0x00000000, 0x00000000, 0x00000000, 0x00000000, 0x40000000 
Basis 3:

0x00000000, 0x00000000, 0x00000000, 0x80000000, 0x00000000, 0x00000000, 0x80000000, 0x00000000, 0x80000000, 0x00000000, 0x00000000, 0x00000000, 0x00000000, 0x00000000, 0x00000000, 0x80000000

Obviously, the input difference of the 30-th step of $C F_{2}^{-1}\left(M \oplus \delta_{2}, C V_{53}\right)$ and $C F_{2}^{-1}\left(M, C V_{53}\right)$ is zero. The bicliques $C_{3}$ is from step 53 to step 58, and the schematic view of the pseudo-preimage attack on 71-step HAS-160 is shown in Fig. 4.

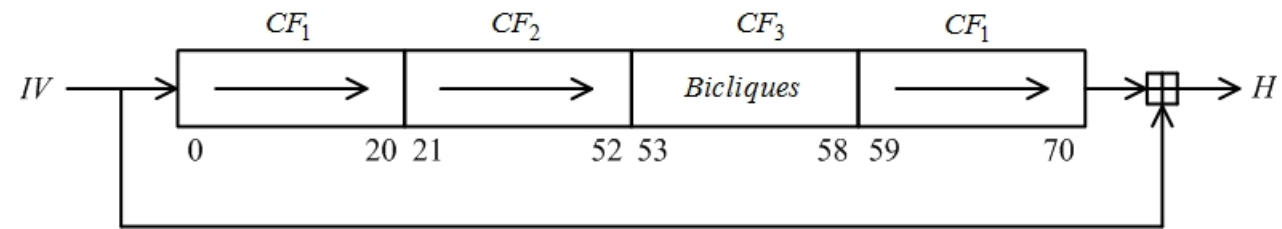

Fig. 4. Schematic view of the pseudo-preimage attack on HAS-160

\subsubsection{Construct 6-step bicliques}

The bicliques which are constructed for $C F_{3}$ cover step 53 to step 58, such that for all $\left(\delta_{1}, \delta_{2}\right) \in L D_{1} \times L D_{2}, C V_{59}\left[\delta_{2}\right]=C F_{3}\left(M \oplus \delta_{1} \oplus \delta_{2}, C V_{53}\left[\delta_{1}\right]\right)$. The parameters used in Section 2.3 are set as follows: $C F_{3}^{1}$ covers step 53 to step 55 and $C F_{3}^{2}$ covers step 56 to step 58, $C V=\left(A_{56}, B_{56}, C_{56}, D_{56}, E_{56}\right)$. For $\delta_{2} \in L D_{2}$ and $\delta_{1} \in L D_{1}$, we can deduce a set of sufficient conditions that make sure $F T\left(\delta_{2}\right)$ and $B T\left(\delta_{1}\right)$ hold. Then the bicliques can be constructed by using the biclique search algorithm in Section 2.3. Note that in the biclique search algorithm, we only set the sufficient conditions in steps 55, 56 and 57 in right branch. By setting those conditions, a biclique can be obtained in a few seconds on a PC. So the complexity of constructing a biclique is negligible. An example of the 6-step biclique is shown in Table 9.

Table 9. An example of the 6-step bicliques in HAS-160

\begin{tabular}{|c|c|c|c|c|c|c|}
\hline word & $m_{1}$ & $m_{6}$ & $m_{8}$ & $m_{10}$ & $m_{15}$ & $m_{0} \oplus m_{9} \oplus m_{2} \oplus m_{11}$ \\
\hline value & 0x33de090e & 0x092798cd & 0x1f5d84c6 & 0xe053d3e1 & 0x00ba96c2 & 0xdcad415a \\
\hline \hline$C V_{53}$ & $A$ & $B$ & $C$ & $D$ & $E$ & \\
\hline value & 0x8fc640fe & 0xd387c000 & 0x1dcc8b3b & 0xcc96b2a0 & 0x7cb3634a & \\
\hline
\end{tabular}

\subsubsection{Search for the mask vector and estimate the error probability}

The mask vector is $T_{42}=(0,0,0 x 86,0,0)$. By extensive experiments, the total test number is $2^{26}$ and the error probability is about 0.429 .

\subsubsection{The attack procedure}

When the bicliques are obtained, we can get $C V_{59}\left[\delta_{2}\right]$ and $C V_{53}\left[\delta_{1}\right]$, and compute $L_{1}=C F_{1}\left(M \oplus \delta_{2}, C V_{59}\left[\delta_{2}\right]\right) \oplus \Delta_{2}$ and $L_{2}=C F_{2}^{-1}\left(M \oplus \delta_{1}, C V_{53}\left[\delta_{1}\right]\right) \oplus \Delta_{1} . \Delta_{1}=\overline{C F_{1}}\left(\delta_{1}, 0\right)$ 
and $\Delta_{2}=\overline{C F_{2}^{-1}}\left(\delta_{2}, 0\right)$, where $C V_{53}, C V_{59}$ and $k_{i}$ are 0 . We need to calculate 18 steps (step 12 to step 29) to retest a candidate pseudo-preimage, $\Gamma_{r e}=18 \Gamma / 71$, and the complexity of constructing a biclique is negligible. Thus, the complexity of the pseudo-preimage attack is $2^{158.13} \approx 2^{160-3} \times(1+18 / 71) /(1-0.429)$.

\subsection{Preimage attack on 71-step HAS-160 from the seventh step}

\subsubsection{Split the compression function and construct the linear spaces}

The forward direction $C F_{1}$ is from step 14 to step 42 and the linear space of this part is $L D_{1}=\left\{\Delta m_{0}\|\ldots\| \Delta m_{15} \mid \Delta m_{0}=\Delta m_{10}=(27,31), \Delta m_{i}=0,0 \leq i \leq 15, i \neq 0,10\right\}$. The basis of $L D_{1}$ is as follows:

\section{Basis 1:}

0x08000000, 0x00000000, 0x00000000, 0x00000000, 0x00000000, 0x00000000, 0x00000000, 0x00000000, 0x00000000, 0x00000000, 0x08000000, 0x00000000, 0x00000000, 0x00000000, 0x00000000, 0x00000000

Basis 2:

0x10000000, 0x00000000, 0x00000000, 0x00000000, 0x00000000, 0x00000000, 0x00000000, 0x00000000, 0x00000000, 0x00000000, 0x10000000, 0x00000000, 0x00000000, 0x00000000, 0x00000000, 0x00000000

Basis 3:

0x20000000, 0x00000000, 0x00000000, 0x00000000, 0x00000000, 0x00000000, 0x00000000, 0x00000000, 0x00000000, 0x00000000, 0x20000000, 0x00000000, 0x00000000, 0x00000000, 0x00000000, 0x00000000

Basis 4:

0x40000000, 0x00000000, 0x00000000, 0x00000000, 0x00000000, 0x00000000, 0x00000000, 0x00000000, 0x00000000, 0x00000000, 0x40000000, 0x00000000, 0x00000000, 0x00000000, 0x00000000, 0x00000000

Basis 5:

0x80000000, 0x00000000, 0x00000000, 0x00000000, 0x00000000, 0x00000000, 0x00000000, 0x00000000, 0x00000000, 0x00000000, 0x80000000, 0x00000000, 0x00000000, 0x00000000, 0x00000000, 0x00000000

In this case, the input difference of the 37-th step of $C F_{1}\left(M \oplus \delta_{1}, C V_{14}\right)$ and $C F_{1}\left(M, C V_{14}\right)$ is zero. The backward direction $C F_{2}^{-1}$ is from step 7 to step 6 connecting with step 76 to step 43 and the linear space of this part is $L D_{2}=\left\{\Delta m_{0}\|\ldots\| \Delta m_{15} \mid \Delta m_{6}=\Delta m_{12}\right.$ $\left.=(27,31), \Delta m_{i}=0,0 \leq i \leq 15, i \neq 6,12\right\}$. The basis of $L D_{2}$ is as follows: 
Basis 1:

0x00000000, 0x00000000, 0x00000000, 0x00000000, 0x00000000, 0x00000000, 0x08000000, 0x00000000, 0x00000000, 0x00000000, 0x00000000, 0x00000000, 0x08000000, 0x00000000, 0x00000000, 0x00000000

Basis 2:

0x00000000, 0x00000000, 0x00000000, 0x00000000, 0x00000000, 0x00000000, 0x10000000, 0x00000000, 0x00000000, 0x00000000, 0x00000000, 0x00000000, 0x10000000, 0x00000000, 0x00000000, 0x00000000

Basis 3:

0x00000000, 0x00000000, 0x00000000, 0x00000000, 0x00000000, 0x00000000, 0x20000000, 0x00000000, 0x00000000, 0x00000000, 0x00000000, 0x00000000, 0x20000000, 0x00000000, 0x00000000, 0x00000000

Basis 4:

0x00000000, 0x00000000, 0x00000000, 0x00000000, 0x00000000, 0x00000000, 0x40000000, 0x00000000, 0x00000000, 0x00000000, 0x00000000, 0x00000000, 0x40000000, 0x00000000, 0x00000000, 0x00000000

Basis 5:

0x00000000, 0x00000000, 0x00000000, 0x00000000, 0x00000000, 0x00000000, 0x80000000, 0x00000000, 0x00000000, 0x00000000, 0x00000000, 0x00000000, 0x80000000, 0x00000000, 0x00000000, 0x00000000

Obviously, the input difference of the 54-th step of $C F_{2}^{-1}\left(M \oplus \delta_{2}, C V_{8}\right)$ and $C F_{2}^{-1}\left(M, C V_{8}\right)$ is zero. The bicliques $C F_{3}$ is from step 8 to step 13, and the schematic view of the preimage attack on 71-step HAS-160 is shown in Fig. 5.

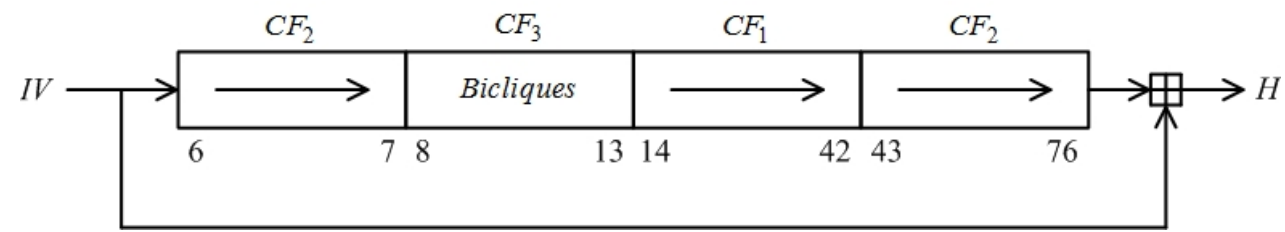

Fig. 5. Schematic view of the preimage attack on HAS-160

\subsubsection{Construct 6-step bicliques}

The bicliques which are constructed for $C F_{3}$ cover step 8 to step 13, such that for all $\left(\delta_{1}, \delta_{2}\right) \in L D_{1} \times L D_{2}, C V_{8}\left[\delta_{2}\right]=C F_{3}\left(M \oplus \delta_{1} \oplus \delta_{2}, C V_{14}\left[\delta_{1}\right]\right)$. The parameters used in Section 2.3 are set as follows: $C F_{3}^{1}$ covers step 8 to step 10 and $C F_{3}^{2}$ covers step 11 to step 13, $C V=\left(A_{11}, B_{11}, C_{11}, D_{11}, E_{11}\right) . F T\left(\delta_{2}\right)$ is deduced as follows: 


$$
\begin{aligned}
& A_{11} \wedge(0 \mathrm{x} 1 \mathrm{f} 0000)=\left(\left(m_{6} \oplus \delta_{2}\right) \wedge(0 \mathrm{xf} 8000000)\right)<<<21, \\
& B_{11} \wedge(0 \mathrm{xf80})=\left(\left(m_{6} \oplus \delta_{2}\right) \wedge(0 \mathrm{xf} 8000000)\right)<<<12, \\
& C_{11} \wedge(0 \mathrm{x} 3 \mathrm{e} 0)=\left(\left(m_{6} \oplus \delta_{2}\right) \wedge(0 \mathrm{xf} 8000000)\right)<<<10 .
\end{aligned}
$$

Condition that satisfies $F T\left(\delta_{2}\right)$ is $D_{11} \wedge(0 \mathrm{xf8000000})=E_{11} \wedge(0 \mathrm{xf8000000}) \cdot \mathrm{BT}\left(\delta_{1}\right)$ is deduced as follows:

$$
C_{11} \wedge(0 \mathrm{xf} 8000000)=\left(\left(m_{0} \oplus \delta_{1}\right) \wedge(0 \mathrm{xf} 8000000)\right) \oplus(0 \mathrm{xf} 8000000) .
$$

Conditions that satisfy $B T\left(\delta_{1}\right)$ are $A_{11} \wedge(0 x f 8000000)=0 x f 8000000, B_{11} \wedge(0 x f 8000000)=$ 0 . Extra condition is $m_{1} \wedge\left(0 \mathrm{xf8000000)}=\left(m_{2} \oplus m_{3}\right) \wedge(0 \mathrm{xf8000000)}\right.$. By setting those conditions, a biclique example can be obtained with probability 1 . So the complexity of constructing a biclique is negligible. An example of the 6-step biclique is shown in Table $\mathbf{1 0 .}$

Table 10. An example of the 6-step bicliques in HAS-160

\begin{tabular}{|c|c|c|c|c|c|c|}
\hline word & $m_{6}$ & $m_{7}$ & $m_{0} \oplus m_{1} \oplus m_{2} \oplus m_{3}$ & $m_{8}$ & $m_{9}$ & $m_{10}$ \\
\hline value & 0x49c1e50b & 0x6c67d9a3 & 0x63a5dd43 & 0xd8b52f39 & 0xbef4c8f3 & 0x39b89099 \\
\hline \hline$C V_{8}$ & $A$ & $B$ & $C$ & $D$ & $E$ & \\
\hline value & 0x8f7b70f3 & 0x873ae2a1 & 0x19f7ee05 & 0xf467e82d & 0xd3fe5704 & \\
\hline
\end{tabular}

\subsubsection{Search for the mask vector and estimate the error probability}

The mask vector is $T_{42}=(0,0 \times 180,0 \times 3,0 \times 2,0)$. By extensive experiments, the total test number is $2^{30}$ and the error probability is about 0.35 .

\subsubsection{The attack procedure}

When the bicliques are obtained, we can get $C V_{8}\left[\delta_{2}\right]$ and $C V_{14}\left[\delta_{1}\right]$, and compute $L_{1}=C F_{1}\left(M \oplus \delta_{2}, C V_{14}\left[\delta_{2}\right]\right) \oplus \Delta_{2}$ and $L_{2}=C F_{2}^{-1}\left(M \oplus \delta_{1}, C V_{8}\left[\delta_{1}\right]\right) \oplus \Delta_{1} . \Delta_{1}=\overline{C F_{1}}\left(\delta_{1}, 0\right)$ and $\Delta_{2}=\overline{C F_{2}^{-1}}\left(\delta_{2}, 0\right)$, where $C V_{8}, C V_{14}$ and $k_{i} 0$. We need to calculate 17 steps (step 37 to step 53) to retest a candidate pseudo-preimage, $\Gamma_{r e}=17 \Gamma / 71$, and the complexity of constructing a biclique is negligible. Thus, the complexity of the pseudo-preimage attack is $2^{155.93} \approx 2^{160-5} \times(1+17 / 71) /(1-0.35)$. Furthermore, this attack can be converted to a preimage attack ( 2 message blocks, with padding) with complexity $2^{158.97}$.

\section{Conclusion}

In this paper, a preimage attack on 34-step RIPEMD-160 with message padding and a pseudo-preimage attack on 71-step HAS-160 without message padding are proposed. The former holds with a complexity of $2^{158.91}$, the latter holds with a complexity of $2^{158.13}$. Furthermore, we locate the linear spaces in another message words and exchange the bicliques construction process and the mask vector search process. A preimage attack on 35-step RIPEMD-160 with complexity $2^{159.38}$ and a preimage attack on 71-step HAS-160 with complexity $2^{158.97}$ are obtained. Both of the attacks satisfy the message padding. Future analysis should be able to explore how to construct bicliques including more steps so that preimage attacks can be implemented on more steps of hash functions. 


\section{References}

[1] Xiaoyun Wang and Hongbo Yu, "How to break MD5 and other hash functions," in Proc. of the 24th Annual International Conference on the Theory and Applications of Cryptographic Techniques, pp. 19-35, May 22-26, 2005. Article (CrossRef Link)

[2] Eli Biham, Rafi Chen, Antoine Joux, Patrick Carribault, Christophe Lemuet and William Jalby, "Collisions of SHA-0 and reduced SHA-1," in Proc. of the 24th Annual International Conference on the Theory and Applications of Cryptographic Techniques, pp. 36-57, May 22-26, 2005. Article (CrossRef Link)

[3] Xiaoyun Wang, Yiqun Lisa Yin and Hongbo Yu, "Finding collisions in the full SHA-1," in Proc. of the 25th Annual International Cryptology Conference, pp. 17-36, August 14-18, 2005. Article (CrossRef Link)

[4] Kazumaro Aoki and Yu Sasaki, "Preimage attacks on one-block MD4, 63-step MD5 and more," in Proc. of the 15th International Workshop on Selected Areas in Cryptography, pp. 103-119, August 14-15, 2008. Article (CrossRef Link)

[5] Deukjo Hong, Bonwook Koo and Yu Sasaki, “Improved preimage attack for 68-step HAS-160,” in Proc. of the 12th International Conference on Information Security and Cryptology, pp. 332-348, December 2-4, 2009. Article (CrossRef Link)

[6] Dmitry Khovratovich, Christian Rechberger and Alexandra Savelieva, "Bicliques for preimages: Attacks on Skein-512 and the SHA-2 family," in Proc. of the 19th International Workshop on Fast Software Encryption, pp. 244-263, March 19-21, 2012. Article (CrossRef Link)

[7] Simon Knellwolf and Dmitry Khovratovich, "New preimage attacks against reduced SHA-1," in Proc. of the 32nd Annual Cryptology Conference, pp. 367-383, August 19-23, 2012. Article (CrossRef Link)

[8] Chiaki Ohtahara, Yu Sasaki and Takeshi Shimoyama, "Preimage attacks on step-reduced RIPEMD-128 and RIPEMD-160," in Proc. of the 6th International Conference on Information Security and Cryptology, pp. 169-186, October 20-24, 2010. Article (CrossRef Link)

[9] Yu Sasaki and Kazumaro Aoki, “A preimage attack for 52-step HAS-160,” in Proc. of the 11th International Conference on Information Security and Cryptology, pp. 302-317, December 3-5, 2008. Article (CrossRef Link)

[10] Yu Sasaki and Kazumaro Aoki, "Finding preimages in full MD5 faster than exhaustive search," in Proc. of the 28th Annual International Conference on the Theory and Applications of Cryptographic Techniques, pp. 134-152, April 26-30, 2009. Article (CrossRef Link)

[11] Jian Guo, San Ling, Christian Rechberger and Huaxiong Wang, "Advanced meet-in-the-middle preimage attacks: First results on full Tiger, and improved results on MD4 and SHA-2," in Proc. of the 16th International Conference on the Theory and Application of Cryptology and Information Security, pp. 56-75, December 5-9, 2010. Article (CrossRef Link)

[12] Whitfield Diffie and Martin E. Hellman, "Exhaustive cryptanalysis of the NBS data encryption standard," Computer, vol. 10, no. 6, pp. 74-84, 1977. Article (CrossRef Link)

[13] Thomas Espitau, Pierre-Alain Fouque and Pierre Karpman, "Higher-order differential meet-in-the-middle preimage attacks on SHA-1 and BLAKE," in Proc. of the 35th Annual Cryptology Conference, pp. 683-701, August 16-20, 2015. Article (CrossRef Link)

[14] Hans Dobbertin, Antoon Bosselaers and Bart Preneel, "RIPEMD-160: A strengthened version of RIPEMD," in Proc. of the 3rd International Workshop on Fast Software Encryption, pp. 71-82, February 21-23 1996. Article (CrossRef Link)

[15] International Organization for Standardization, "Information technology - Security techniques Hash-functions - Part 3: Dedicated hash functions (2004),” ISO/IEC 10118-3:2004, Article (CrossRef Link).

[16] Florian Mendel, Norbert Pramstaller, Christian Rechberger and Vincent Rijmen, "On the collision resistance of RIPEMD-160," in Proc. of the 9th International Conference on Information Security, pp. 101-116, August 30 - September 2, 2006. Article (CrossRef Link)

[17] Florian Mendel, Tomislav Nad, Stefan Scherz and Martin Schläffer, "Differential attacks on reduced RIPEMD-160," in Proc. of the 15th International Conference on Information Security, pp. 
23-38, September 19-21, 2012. Article (CrossRef Link)

[18] Florian Mendel, Thomas Peyrin, Martin Schläffer, Lei Wang and Shuang Wu, "Improved cryptanalysis of reduced RIPEMD-160," in Proc. of the 19th International Conference on the Theory and Application of Cryptology and Information Security, pp. 484-503, December 1-5, 2013. Article (CrossRef Link)

[19] Yu Sasaki and Lei Wang, "Distinguishers beyond three rounds of the RIPEMD-128/-160 compression functions," in Proc. of the 10th International Conference on Applied Cryptography and Network Security, pp. 275-292, June 26-29, 2012. Article (CrossRef Link)

[20] Telecommunications Technology Association, "Hash Function Standard Part 2: Hash Function Algorithm Standard, HAS-160 (2000),” TTAS.KO-12.0011/R2. Article (CrossRef Link).

[21] Aaram Yun, Soo Hak Sung, Sangwoo Park, Donghoon Chang, Seokhie Hong and Hong-Su Cho, "Finding collision on 45-step HAS-160," in Proc. of the 8th International Conference on Information Security and Cryptology, pp. 146-155, December 1-2, 2005. Article (CrossRef Link)

[22] Xiaoyun Wang, Xuejia Lai, Dengguo Feng, Hui Chen and Xiuyuan Yu, "Cryptanalysis of the hash functions MD4 and RIPEMD,” in Proc. of the 24th Annual International Conference on the Theory and Applications of Cryptographic Techniques, pp. 1-18, May 22-26, 2005. Article (CrossRef Link)

[23] Hong-Su Cho, Sangwoo Park, Soo Hak Sung and Aaram Yun, “Collision search attack for 53-step HAS-160,” in Proc. of the 9th International Conference on Information Security and Cryptology, pp. 286-295, November 30 - December 1, 2006. Article (CrossRef Link)

[24] Florian Mendel and Vincent Rijmen, "Colliding message pair for 53-step HAS-160,” in Proc. of the 10th International Conference on Information Security and Cryptology, pp. 324-334, November 29-30, 2007. Article (CrossRef Link)

[25] Florian Mendel, Tomislav Nad and Martin Schläffer, "Cryptanalysis of round-reduced HAS-160," in Proc. of the 14th International Conference on Information Security and Cryptology, pp. 33-47, November 30 - December 2, 2011. Article (CrossRef Link)

[26] Aleksandar Kircanski, Riham AlTawy and Amr M. Youssef, "Heuristic for finding compatible differential paths with application to HAS-160," in Proc. of the 19th International Conference on the Theory and Application of Cryptology and Information Security, pp. 464-483, December 1-5, 2013. Article (CrossRef Link)

[27] Alfred J. Menezes, Paul C. van Oorschot and Scott A. Vanstone, Handbook of Applied Cryptography, CRC Press, 1996.

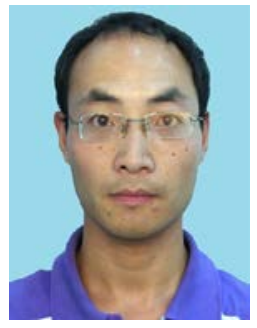

Yanzhao Shen received the B.S. degree in School of Computer and Information Engineering form Henan University, Kaifeng, China, M.S. in School of Computer Science and Technology from Donghua University, Shanghai, China. Now he is a Ph.D student in School of Mathematics, Shandong University. His current research interests include cryptography, computer and network security.

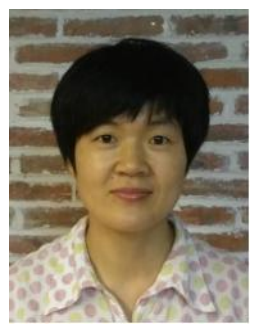

Gaoli Wang received the B.S. degree in Fundamental Mathematics from Shandong University, Jinan, China, M.S. and Ph.D degree in Information Security from Shandong University, Jinan, China. She is currently an assistant professor at School of Computer Science and Software Engineering, East China Normal University. Her research interests include cryptography, computer and network security. 